\title{
Traces of fatigue in an attention task
}

\author{
E. SOETENS, J. HUETING, and F. WAUTERS \\ University of Brussels, Brussels, Belgium
}

\begin{abstract}
The present study is one in a series undertaken to analyze the influence of physical fatigue on a visual perception task. Thirty minutes after being exhausted on a bicycle ergometer, 20 subjects executed a visual perception task involving tachistoscopically presented random groups of 3 to 12 dots. The subjects responded by reporting the number of dots, and their response times were measured. Under the influence of fatigue, the number of correct responses decreased, especially for the larger groups of dots. No significant differences in response times were found between fatigued and nonfatigued subjects. We conclude that fatigued subjects avoid attention that demands controlled processing and tend to adhere to uniform automatic processing.
\end{abstract}

It is intuitively felt that physical fatigue has an influence on performance of a subsequent mental task. However, there is no agreement on how this influence interferes in information processing. The present study is a follow-up experiment in a series undertaken to analyze the nature of the influence of fatigue on a visual perception task.

Research into the influence of physical effort on cognitive processes is rather confused. In a meta-analysis of the literature, Tomporowski and Ellis (1986) concluded that clear data were still missing. Positive, negative, as well as no influence of physical fatigue on information processing have been reported. There are several reasons for these discrepancies, ranging from sloppy methodology to differences in induced fatigue level, the timing between physical and subsequent mental tasks, and differences in cognitive tasks.

Nevertheless, there is general agreement that some central cognitive mechanisms are sensitive to physical fatigue. Support for this conjecture can be found in investigations using EMG measures to fractionate reaction time (RT) (Hanson \& Lofthus, 1978).

It is improbable that this influence is caused by a lack of energy supply. It has been demonstrated that the energy consumption of the brain is practically invariable, regardless of whether the subject under investigation is sleeping, resting, or solving a complex problem (Schmidt, 1983). What then are the underlying processes that might explain the deterioration, if any, of mental performance under the influence of physical effort?

It could be that deterioration of cognitive performance under the influence of fatigue is caused by a shift in activation from higher cognitive to lower functions. Physiological measurements indicate that the distribution of energy in the brain can change under the influence of mental processes (e.g., Donchin, 1981; Ingvar, 1976; Posner, Petersen, Fox, \& Raichle, 1988). However, comparison of these

\footnotetext{
Requests for reprints should be sent to Eric Soetens, Laboratory of Experimental Psychology, University of Brussels, Waversesteenweg 1077, B-1160 Brussels, Belgium.
}

measurements between fatigued and nonfatigued subjects on the same task have not been carried out heretofore.

Several investigations support the conjecture that tired subjects avoid complex decisions. Holding (1974), for example, found that these subjects opted for uniform, less demanding working procedures, although they knew that the more demanding procedures were faster and more accurate. Similar results were observed in real-life situations. Rasmussen and Jensen (1974) reported a change in error-detecting strategy for a group of workers in an electronics firm, going from complex attention-demanding decision processes, leading to fast and accurate solutions, to a series of simple yes-no decisions, which were often redundant.

A theoretical framework separating higher level, attentiondemanding processes from lower level, automatic processes has been developed by Schneider and Shiffrin (Schneider, Dumais, \& Shiffrin, 1984; Schneider \& Shiffrin, 1977; Shiffrin \& Schneider, 1977). According to these authors, automatic processes are fast, fairly effortless, and responsible for the performance of well-developed skilled behavior. In contrast, controlled processes are effortful and capacity limited, and are required under novel and strongly varying conditions. In terms of Schneider's theory, fatigue influence could be explained by a gradual switch from controlled to automatic processes. It follows that components needing controlled processing will be disturbed.

Support for a differential influence of fatigue on automatic and attention-demanding processing mechanisms can be found in our previous experiments (Soetens, Deboeck, \& Hueting, 1984; Vervaeck, Deboeck, Hueting, \& Soetens, 1982), in which subjects performed an exhausting physical task, followed by a dual, paced visualauditory task. In those tasks, subjects reported the number of tachistoscopically presented random groups of dots while simultaneously reporting a random series of low and high tones.

No difference in performance was found between physically fresh and fatigued subjects, either for the auditory task or for presentations of groups of 3-7 dots in the visual perception task. In the more difficult part of the visual 
task, however, that involving presentations of groups of 8-12 dots, there was a deteriorating effect due to fatigue. This effect was visible only in terms of the number of omitted responses, that is, those trials where the subject was not able to respond to the presented stimulus.

The results suggest that subjects first form a quick and global impression of the stimulus array. With this tentative information, they evaluate the complexity of the task, on the basis of which they decide to continue or to stop processing. Presumably, the number of interruptions increases under the influence of fatigue due to an increased tendency for the subject to avoid the higher level, attentiondemanding processes.

In the present visual perception task, the stimuli were dot patterns similar to those used in our previous studies (Soetens et al., 1984; Vervaeck et al., 1982). Care was taken to avoid the presentation of recognizable patterns (Van Oeffelen \& Vos, 1982), so that only the number of dots would determine stimulus complexity.

In the present research, responses are made compulsory in an attempt to shift the influence of fatigue from omitted responses to the number of correct responses, which will make for a more substantial database. A differential influence of fatigue on small versus large numbers of dots could reflect two different processing mechanisms. According to Klahr (1973), the processes that dominate the visual perception system differ depending on the complexity of the presented dot pattern. The dominating process for small numbers of dots, say up to 5 or 6 , is called subitizing. Klahr describes the process as a serial self-terminating scan of short-term memory for a match between the encoded stimulus and a set of quantitative symbols. Subitizing is assumed to be an automatic process, demanding little attention. For larger numbers, the dots have to be counted, which is a more complex process that demands more attention. The counting process is a controlled process and should be more liable to be avoided in conditions of increased fatigue.

Besides the number of correct responses, RT is also measured. It can be expected that the RT data for small numbers will conform to the slope of the subitizing process as reported by Klahr (1973). For larger numbers, however, because of the short exposure duration, subjects will not have the opportunity to complete the more complex processing. The increased slope that is usually found for the RT curve of the counting process will accordingly be replaced by a flattening of the curve as a result of the important contribution made by the number of guessed at responses.

Intuitively, one would expect RT to slow down when subjects are tired. However, other studies have demonstrated that the relationship between fatigue and RT is more complex (e.g., Kahneman \& Treisman, 1984; Welford, 1977). In our dual-task studies (Soetens et al., 1984; Vervaeck et al., 1982), we found that fatigued subjects gave up the processing of only the complex stimuli. Now that subjects were to be obliged to respond, they would probably reply more readily, even when the processing of the stimulus was not complete. The outcome should be a more pronounced flattening of the RT curve for the larger numbers of dots. In general, we can expect fatigued subjects to respond in a uniform way, relying predominantly on automatic mechanisms and avoiding attentiondemanding, controlled processing.

\section{METHOD}

\section{Subjects}

Twenty 20- to 24-year-old students, 15 male and 5 female, participated in the experiment. None were active in sports, and none had had previous experience with tachistoscopic experiments.

\section{Physical Task}

Subjects had to cycle for $30 \mathrm{~min}$ on a bicycle ergometer at a constant rate of $50 \mathrm{rpm}$. The loads were $50 \mathrm{~W}$ during the initial $10 \mathrm{~min}, 100 \mathrm{~W}$ during the next $10 \mathrm{~min}, 125 \mathrm{~W}$ during the next $5 \mathrm{~min}$, and $150 \mathrm{~W}$ during the last $5 \mathrm{~min}$. The average heart rate at the end of the physical task was $154 \mathrm{beats} / \mathrm{min}$ with a standard deviation of 13.5 .

\section{Visual Attention Task}

The stimuli were 3 to 12 black dots on white cards, which were presented by means of a three-field Electronic Development tachistoscope. Care was taken to spread the dots systematically over the surface. The dots appeared in the same amount on each location. Recognizable patterns were avoided as much as possible by randomizing the dot locations (Soetens et al., 1984). The maximum visual angle from the fixation point was $2.5^{\circ}$.

RT was the number of milliseconds between the beginning of stimulus presentation and the beginning of the response. The response was registered by means of a voice key.

\section{Procedure}

After execution of the physical task, the subjects were given a 30-min pause during which they had the opportunity to wash and refresh themselves. The subjects were then seated in front of the three-field tachistoscope, with their heads fitting comfortably into a viewing hood.

A fixation point, clearly distinguishable from the target dots, was presented before each trial. The subjects initiated each trial by pushing a button. A trial consisted of the 50 -msec presentation of a card with a group of dots, followed by a dark interstimulus interval of $100 \mathrm{msec}$ and then the presentation of a mask for $50 \mathrm{msec}$. The mask card displayed 24 dots, that is, a dot at each possible target location. The subjects were instructed to answer as accurately and as quickly as possible. They were informed that the minimum number of dots was 3 and the maximum number, 12 . RTs were recorded, and the experimenter noted the answers. Two groups of 10 subjects participated in two sessions, separated by a 1-week interval. A session lasted approximately $15 \mathrm{~min}$ and consisted of 160 trials. A crossover design was used such that one group was put through the physical task before performing the visual attention task, while the other group was not. In the second session, 1 week later, the conditions were reversed.

\section{RESULTS AND DISCUSSION}

A general analysis of variance (ANOVA) with repeated measures on the number of correct responses showed a significant main effect of fatigue $[F(1,18)=10.51, p<$ $.01]$. The introduction of compulsory responses clearly had the intended effect. The marginal effect of fatigue on the number of omitted responses found in our previous research was replaced by a significant effect in the number of correct responses.

The ANOVA further showed an interaction between fatigue and number of dots $[F(9,162)=2.52, p<.01]$, supporting the results of our earlier research (Soetens et al., 1984; Vervaeck et al., 1982). The present inter- 


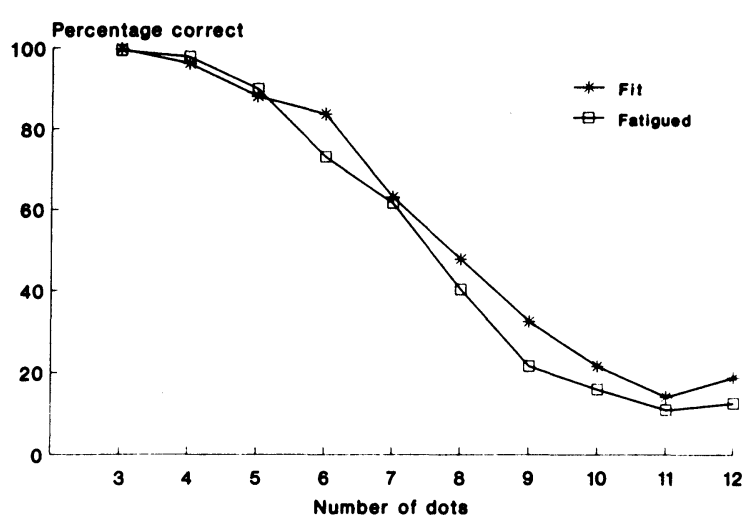

Figure 1. Percentage of correct responses as a function of number of dots for fatigued and nonfatigued (nt) subjects.

action indicated again that the influence of fatigue was confined to the larger numbers of dots. Figure 1, showing the number of correct responses as a function of number of dots, supports this presumption. The differences between fatigued and nonfatigued subjects are mainly limited to stimuli with eight or more dots (the six-dot stimuli also shows a substantial difference).

As in our previous studies, we carried out two separate ANOVAs on the number of correct responses for the 3-7 and 8-12 dot patterns. No significant effect of fatigue was found for the smaller numbers of dots $[F(1,18)=$ 3.14], although a slight trend was found $(p=.09)$. The automatic subitizing process, which is supposed to be the main processing method for these dot quantities, was only slightly influenced by fatigue.

A clearly significant fatigue effect was found with the larger numbers of dots $[F(1,18)=7.03, p<.016]$. According to Klahr (1973), counting forms an important part of the processing method for these dot quantities. The counting process thus seems to be significantly influenced by the introduction of fatigue.

The course of the RT curve as a function of number of dots, shown in Figure 2, was as predicted. For the smaller number of dots, 3 to 8 , the curve has a slope of .38; for the higher number of dots, the curve flattens out

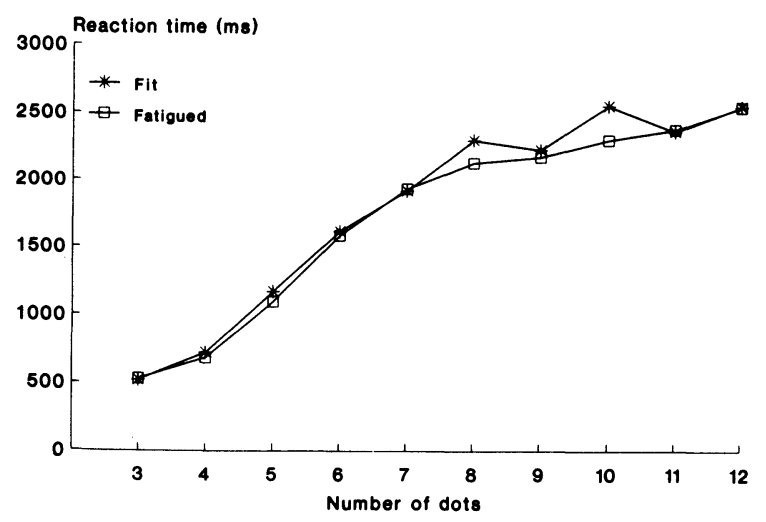

Figure 2. Median reaction times as a function of number of dots for fatigued and nonfatigued (fit) subjects. to a slope of .075 . The extra flattening of the curve predicted for the influence of fatigue is only marginal. An ANOVA on the RT data showed no main effect of fatigue $[F(1,18)=.85]$ and no significant interaction with the number of dots. Thus, the RT data do not support the notion of the avoidance of a complex processing mechanism. The reason for the absence of a fatigue effect in the RT data probably lies in the method of stimulus presentation. An immediate consequence of the short stimulus presentation is the flattening of the RT curve for the larger numbers of dots instead of an increase in slope, reflecting the counting mechanism. This flattening is possibly due to the subjects' not having enough time to finish the more time-consuming counting process even in the nonfatigued condition. In this way, the influence of fatigue on the counting process is limited, and does not become evident in the RT data. Nevertheless, the short time that controlled processes are active is long enough to differentiate between fatigued and nonfatigued subjects in terms of the number of correct responses.

The fatigue influence can easily be incorporated into Schneider and Shiffrin's (1977) two-process theory of human information processing. The automatic process in this case is subitizing. The association of small groups of dots with numbers is obviously a well-learned sequence of elements, proceeding automatically and uninfluenced by capacity limitations of the system (Schneider \& Shiffrin, 1977).

The controlled processing mechanism is counting. It is characterized as slow, effortful, and capacity limited, and as being under the subject's control and most likely to be influenced by fatigue. The contribution of controlled processes explains the increased influence of fatigue on the larger numbers of dots.

Our previous experiments (Soetens et al., 1984; Vervaeck et al. 1982) show an abrupt increase of omitted responses between 7 and 8 dots, suggesting an abrupt change in processing method. The present data, however, support a gradual transition. There is already a trend toward significance for the influence of fatigue on the smaller numbers of dots. It could be that some controlled processes are already at work in a limited way for the smaller quantities. According to Schneider (Schneider et al., 1984), most tasks involve a mixture of automatic and controlled processes. The more complex the task, the larger the share of controlled processes.

The influence of fatigue on automatic and controlled processes needs further investigation. One approach would be to return to Klahr's (1973) original design, with subject-paced stimulus exposure time. The slopes of the RT curves for the different processing mechanisms can then be analyzed for fatigued and nonfatigued subjects. Another approach would be to try to explain the absence of a fatigue influence on RT while the number of correct responses decreases significantly. Subjects obviously shift to a more lax criterion. Further investigation into the influence of fatigue on speed-accuracy functions can possibly lead to an explanation in terms of automatic and controlled processes. 


\section{REFERENCES}

Donchin, E. (1981). Surprise! ... Surprise? Psychophysiology, 18, 493-513.

Hanson, C., \& Lofthus, G. K. (1978). Effects of fatigue and laterality on fractionated reaction time. Journal of Motor Behavior, 10, 177-184.

Holding, D. H. (1974). Risk, effort, and fatigue. In M. G. Wade \& R. Martens (Eds.), Psychology of motor behavior and sport. Urbana, IL: Human Kinetics Publications.

INGVAR, D. H. (1976). Functional landscapes of the dominant hemisphere. Brain Research, 107, 181-197.

Kahneman, D., \& Treisman, A. (1984). Changing views of attention and automaticity. In R. Parasuraman \& D. R. Davies (Eds.), Varieties of attention (pp. 29-61). Orlando, FL: Academic Press.

KLAHR, D. (1973). Quantification processes. In W. G. Chase (Ed.), Visual information processing (pp. 3-34, 527-546). New York: Academic Press.

Posner, M. I., Petersen, S. E., Fox, P. T., \& Raichle, M. E. (1988). Localization of cognitive operations in the human brain. Science, 240 , 1627-1631.

RASMUSSEN, J., \& JENSEN, A. (1974). Mental procedures in real-life tasks, a case study of electronic trouble shooting. Ergonomics, 17, 293-307.

SCHMIDT, R. F. (1983). Integrative functions of the central nervous system. In R. F. Schmidt \& G. Thews (Eds.), Human physiology (2nd ed., pp. 124-165). Berlin: Springer-Verlag.
Schneider, W., Dumais, S. T., Shiffrin, R. M. (1984). Automatic and controlled processing and attention. In $R$. Parasuraman \& $D$. R. Davies (Eds.), Varieties of attention (pp. 1-27). Orlando, FL: Academic Press.

SChNeIDER, W., \& ShIFFrin, R. M. (1977). Controlled and automatic human information processing: I. Detection, search, and attention. Psychological Review, 84, 1-66.

SHIFFrIN, R. M., SCHNEIDER, W. (1977). Controlled and automatic human information processing: II. Perceptual learning, automatic attending, and a general theory. Psychological Review, 84, 127-190.

Soetens, E., Deboeck, M., Hueting, J. (1984). Traces of fatigue in an attention dual task: II. Bulletin of the Psychonomic Society, 22, 514-516.

Tomporowski, P. D., Ellis, N. R. (1986). The effects of exercise on cognitive processes: A review. Psychological Bulletin, 99, 338-346.

Vervaeck, K., Deboeck, M., Hueting, J., \& Soetens, E. (1982). Traces of fatigue in an attention dual task. Bulletin of the Psychonomic Society, 19, 151-154.

Van Oefrelen, M. P., \& Vos, P. G. (1982). Configurational effects on the enumeration of dots: Counting by groups. Memory \& Cognition, 10, 396-404.

WELPORD, A. T. (1977). Serial reaction times, continuity of task, singlechannel effects, and age. In S. Dornic (Ed.), Attention and performance VI (pp. 79-97). Hillsdale, NJ: Erlbaum.

(Manuscript received June 25, 1991.) 